\title{
Detección molecular de Rickettsia typhi en perros de una comunidad rural de Yucatán, México
}

\author{
Daly Martínez-Ortiz¹, Marco Torres-Castro², Edgar Koyoc-Cardeña ${ }^{3}$, Karina López²,

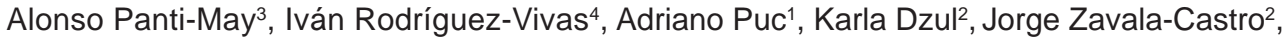 \\ Anuar Medina-Barreiro ${ }^{3}$, Juan Chablé-Santos ${ }^{5}$, Pablo Manrique-Saide ${ }^{3,5}$ \\ Coordinación de Zoonosis, Servicios de Salud de Yucatán, Mérida, México \\ 2 Laboratorio de Enfermedades Emergentes y Reemergentes, Centro de Investigaciones Regionales "Dr. Hideyo \\ Noguchi", Universidad Autónoma de Yucatán, Mérida, México \\ 3 Unidad Colaborativa para Bioensayos Entomológicos, Campus de Ciencias Biológicas y Agropecuarias, \\ Universidad Autónoma de Yucatán, Mérida, México \\ 4 Laboratorio de Parasitología, Campus de Ciencias Biológicas y Agropecuarias, Universidad Autónoma de \\ Yucatán, Mérida, México \\ 5 Departamento de Zoología, Campus de Ciencias Biológicas y Agropecuarias, Universidad Autónoma de \\ Yucatán, Mérida, México
}

Introducción. Rickettsia typhi es la bacteria causante del tifus múrido o endémico, el cual es transmitido al ser humano principalmente por medio de las heces infectadas de pulgas y en cuyo ciclo de infección se encuentran involucrados distintos animales sinantrópicos y domésticos. En la comunidad rural de Bolmay, Yucatán, México, se reportaron casos de tifus múrido en seres humanos durante el periodo 2007-2010.

Objetivo. Identificar la presencia de R. typhi y estimar la frecuencia de infección en perros de Bolmay, México.

Materiales y métodos. Se tomaron muestras de sangre completa de 128 perros, se les extrajo el ADN total y se analizaron mediante reacción en cadena de la polimerasa (PCR) para amplificar los fragmentos del gen de $17 \mathrm{kDa}$ y omp $B$, y confirmar la presencia de Rickettsia spp. Los productos de las reacciones se enviaron a secuenciación y se les hizo un análisis de alineamiento con Basic Local Alignment Search Tool (BLAST).

Resultados. Se encontró una frecuencia de infección de 5,5\% (7/128). El alineamiento demostró $99 \%$ de homologación para el gen de 17 kDa y $100 \%$ para el gen omp B en $R$. typhi.

Conclusión. Se detectó la presencia de $R$. typhi pero una baja frecuencia de infección en perros de la comunidad de estudio; sin embargo, la especie podría representar un riesgo de transmisión para los seres humanos.

Palabras clave: Rickettsia typhi, perros, México.

doi: http://dx.doi.org/10.7705/biomedica.v36i2.2913

Molecular evidence of Rickettsia typhi infection in dogs from a rural community in Yucatán, México

Introduction: Rickettsia typhicauses murine or endemic typhus, which is transmitted to humans primarily through flea bites contaminated with feces. Synanthropic and domestic animals also contribute to the infection cycle of $R$. typhi. Cases of murine typhus in humans were reported in the rural community of Bolmay, Yucatán, México, between 2007 and 2010.

Objective: To identify the presence of $R$. typhi and estimate the frequency of infection in dogs from Bolmay, México, a locality with previous reports of murine typhus in humans.

Materials and methods: Whole blood samples were taken from 128 dogs. Total DNA was extracted for use in the polymerase chain reaction (PCR) to amplify fragments of the $17 \mathrm{kDa}$ and omp $B$ genes and confirms the presence of Rickettsia spp. The reaction products were sequenced, and alignment analysis was performed using the BLAST tool.

\footnotetext{
Contribución de los autores:

Daly Martínez-Ortiz: diseño del estudio, muestreo y coordinación de actividades de campo, concepción y revisión del manuscrito final Marco Torres-Castro: diagnóstico molecular, interpretación de resultados, concepción y revisión del manuscrito final Edgar Koyoc-Cardeña: muestreo de campo, interpretación de resultados, concepción y revisión del manuscrito final Karina López: diagnóstico molecular e interpretación de resultados Alonso Panti-May: muestreo de campo y revisión del manuscrito final Iván Rodríguez-Vivas, Jorge Zavala-Castro y Karla Dzul: interpretación de resultados, concepción y revisión del manuscrito final Adriano Puc y Anuar Medina-Barreiro: muestreo de campo Juan Chablé-Santos: interpretación de resultados y revisión del manuscrito final

Pablo Manrique-Saide: coordinación general de actividades, interpretación de resultados, revisión del manuscrito final
} 
Results: The frequency of $R$. typhi infection in dogs was $5.5 \%(7 / 128)$. The alignment identified $99 \%$ and $100 \%$ homology to the $R$. typhi $17 \mathrm{kDa}$ and omp $B$ genes, respectively.

Conclusion: We confirmed the presence of $R$. typhi in dogs in the studied community but at a low frequency. However, there is potential risk of transmission to humans.

Key words: Rickettsia typhi, dogs, México

doi: http://dx.doi.org/10.7705/biomedica.v36i2.2913

Las rickettsiosis son ocasionadas por bacterias intracelulares estrictas Gram negativas, pertenecientes al género Rickettsia, familia Rickettsiaceae, orden Rickettsiales (1), que se consideran un problema emergente de salud pública, principalmente en países de América Latina (2). El género se divide en dos grupos según el espectro de manifestaciones clínicas que ocasionan: a) fiebre manchada y b) tifus, los cuales son transmitidos por artrópodos vectores como las garrapatas, las pulgas y los piojos a diversos vertebrados domésticos y silvestres, incluido el ser humano (3-5).

En el grupo que ocasiona el tifus se encuentra la especie Rickettsia typhi, causante del tifus múrido o endémico, en cuyo ciclo clásico de infección participan huéspedes mamíferos (roedores y seres humanos) y vectores (pulgas). La infección se transmite al ser humano principalmente por medio de las heces de pulgas infectadas $(3,4)$. También se conoce un ciclo en el que pueden intervenir otros animales domésticos y silvestres, como gatos, zarigüeyas y perros, considerados como huéspedes o reservorios, aunque poco eficientes, ya que el periodo de circulación de las rickettsias en la sangre después de la infección es breve (6).

La circulación de $R$. typhi en perros se ha reportado en varios países; sin embargo, la información basada en pruebas diagnósticas directas es escasa (7). La convivencia con perros y animales de compañía se ha asociado a la presentación de casos de rickettsiosis y de tifus múrido en seres humanos $(7,8)$, pues actúan como vehículos de los vectores infectados (9), por lo que su papel en el ciclo infeccioso de estas enfermedades puede ser determinante (10).

En Yucatán, México, se han documentado casos de tifus múrido en seres humanos (11-13) y, aunque se ha encontrado $R$. typhi en muestras

Correspondencia:

Pablo Manrique, Carretera Mérida-X'matkuil, Kilómetro 15.5, Mérida, México

Teléfono: (52-999) 9423200

msaide@correo.uady.mx

Recibido: 15/07/15; aceptado: 09/09/15 de sangre de Rattus rattus en la región (14), aún no se han determinado otros posibles reservorios u huéspedes involucrados en el ciclo natural de la infección.

El objetivo del presente estudio fue determinar y estimar la frecuencia de infección de $R$. typhi mediante métodos moleculares en perros de una comunidad rural de Yucatán, México, con antecedentes de tifus múrido en la población humana.

\section{Materiales y métodos}

\section{Área de estudio}

El estudio se llevó a cabo en la comunidad rural de Bolmay, Yucatán, México (20³2'56" N, 8756'04" O) (figura 1), que tiene una población de 312 habitantes distribuidos en 60 viviendas construidas en su mayoría con materiales desechables o perecederos. El clima de la región es cálido subhúmedo (Aw 0), con lluvias en verano, una temperatura media anual de $32{ }^{\circ} \mathrm{C}$ y vegetación de tipo selva baja caducifolia (15).

\section{Recolección de muestras}

La muestra incluyó a todos los perros de la comunidad; se extrajeron 3 a $5 \mathrm{ml}$ de sangre completa de cada perro por punción de la vena cefálica (previa asepsia del área). Las muestras se recolectaron en tubos BD estériles (Vacutainer ${ }^{\mathrm{TM}}$

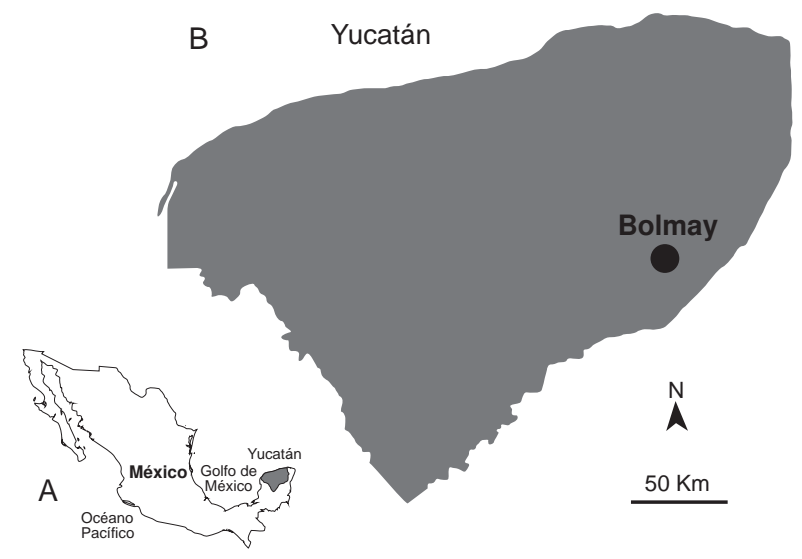

Figura 1. Localización de Yucatán (área sombreada) A. Ubicación de Yucatán en el mapa de México. B. Mapa de Yucatán que muestra el sitio de estudio (punto oscuro) 
Blood Collection Tube, Franklin Lakes, NJ, Estados Unidos) que contenían ácido etilen-diaminotetraacético (EDTA), etiquetados con los códigos de identificación de cada perro. Las muestras de sangre se refrigeraron a $4{ }^{\circ} \mathrm{C}$ hasta su procesamiento en el Laboratorio de Enfermedades Emergentes y Reemergentes del Centro de Investigaciones Regionales "Dr. Hideyo Noguchi" de la Universidad Autónoma de Yucatán. Además, se registraron los datos de sexo, procedencia, raza y edad (joven, adulto o de edad avanzada) de cada perro. Las muestras fueron recolectadas por médicos veterinarios durante marzo de 2014.

\section{Extracción del ADN}

Las muestras de sangre obtenidas de los perros se procesaron para la extracción de ADN con el kit comercial DNeasy Blood and Tissue QIAGEN (QIAGEN ${ }^{\circledR}$, Valencia, CA, Estados Unidos), siguiendo las especificaciones del fabricante. El ADN total extraído $(100 \mu \mathrm{l})$ se cuantificó en un espectrofotómetro (NanoDrop2000 ${ }^{\circledR}$, Thermo Scientific, Waltham, MA, Estados Unidos), y se conservó a $-20{ }^{\circ} \mathrm{C}$ hasta su posterior uso en las pruebas de diagnóstico molecular.

\section{Diagnóstico molecular de Rickettsia spp.}

El diagnóstico molecular se hizo mediante reacción en cadena de la polimerasa (PCR) anidada, con el objetivo de amplificar el gen de la proteína de superficie htrA en su segmento de 17 kDa (antígeno), porción muy conservada en microorganismos pertenecientes al género Rickettsia (16). En la primera reacción se emplearon los cebadores propuestos por Webb, et al., (17): 17kD1 (5'-GCTCTTGCAACTTCTATGTT-3') y 17kD2 (5'-CATTGTTCGTCAGGTTGGCG-3'), los cuales amplifican un segmento de $434 \mathrm{pb}$ del gen de $17 \mathrm{kDa}$. En la segunda reacción se emplearon los cebadores descritos por Schriefer, et al. (18): 17kN1 (5'-CATTACTTGGTTCTCAATTCGGT-3') y $17 \mathrm{kN2}$ (5'-TTTTATTAGTGGTTACGTAA-3'), que amplifican un segmento de $232 \mathrm{pb}$.

Ambas reacciones se hicieron bajo las siguientes condiciones de amplificación: desnaturalización inicial a $95{ }^{\circ} \mathrm{C}$ por cinco minutos, seguida de 35 ciclos a $94{ }^{\circ} \mathrm{C}$ por 30 segundos, a $58^{\circ} \mathrm{C}$ durante 45 segundos, a $72{ }^{\circ} \mathrm{C}$ por un minuto, y una extensión final a $72^{\circ} \mathrm{C}$ durante siete minutos. Los reactivos utilizados en las dos reacciones tenían las siguientes concentraciones: solución tampón PCR 10X, 2,5 mM de $\mathrm{MgCl}_{2}, 10 \mathrm{mM}$ de mezcla del dNTP, $10 \mu \mathrm{M}$ de cada cebador, 1,25 U de Taq ADN polimerasa recombinante (Thermo Scientific ${ }^{\mathrm{TM}}$, Waltham, MA, Estados Unidos) y $150 \mathrm{ng} / \mu \mathrm{l}$ de ADN, para un volumen final de $25 \mu$ l. En cada reacción se utilizaron un control positivo (plásmido de Rickettsia parkeri) y uno negativo (agua estéril). Los productos amplificados se analizaron por electroforesis en geles verticales de poliacrilamida al $8 \%$ teñidos con bromuro de etidio y se visualizaron con ayuda de un fotodocumentador (Bio-Rad ${ }^{\circledR}$, Hercules, CA, Estados Unidos).

El diagnóstico molecular de Rickettsia spp. se complementó mediante una PCR estándar (punto final) que amplificó un fragmento del gen de la proteína de membrana externa $\mathrm{B}$ (omp $B$ ), común en microorganismos pertenecientes a este género. En esta reacción se emplearon los cebadores descritos por Peniche-Lara, et al. (19): Rp.330(2) (5'-ATGGCTTCAAAAACCAAATTTTCTAA-3') y Rp.330(2) (5'-GCTCTACCTGCTCCATTATCTGT ACC-3'), los cuales amplifican una región de 990 a 1.000 pb. Las condiciones de amplificación de la reacción fueron las siguientes: desnaturalización inicial a $95{ }^{\circ} \mathrm{C}$ por cinco minutos, seguida de 35 ciclos a $94{ }^{\circ} \mathrm{C}$ por 30 segundos, a $58{ }^{\circ} \mathrm{C}$ por 45 segundos, a $72^{\circ} \mathrm{C}$ durante dos minutos, y una extensión final a $72{ }^{\circ} \mathrm{C}$ durante 10 minutos. Los reactivos se utilizaron con las siguientes concentraciones: solución tampón PCR 10X, 2,5 $\mathrm{mM}$ de $\mathrm{MgCl}_{2}, 10 \mathrm{mM}$ de mezcla del dNTP, 10 $\mu \mathrm{M}$ de cada cebador, 1,25 U de ADN polimerasa Taq recombinante (Thermo Scientific ${ }^{\top M}$, Waltham, MA, Estados Unidos), con un volumen final de $25 \mu$ l. Los controles positivo y negativo, y la visualización de los productos, fueron iguales a los descritos para la reacción anidada.

\section{Secuenciación}

Se secuenciaron tres amplicones de cada muestra positiva en el Instituto de Biotecnología de la Universidad Nacional Autónoma de México. Las secuencias obtenidas se alinearon con secuencias homólogas de otras especies de rickettsias depositadas en el GenBank ${ }^{\circledR}$ mediante el programa Basic Local Alignment Search Tool (BLAST) en el National Center for Biotechnology Information (Estados Unidos) (http://blast.ncbi.nlm.nih.gov/ Blast.cgi), usando el algoritmo megablast.

\section{Análisis estadístico}

Las variables de los perros muestreados y los resultados de la PCR se analizaron con estadística descriptiva. Además, se usó una prueba de ji al cuadrado para establecer la asociación (IC ${ }_{95 \%}$, 
$p<0,05$ ) entre las variables independientes (sexo, procedencia, raza y edad) y los resultados de la PCR (variable dependiente). Los datos se analizaron con ayuda del programa PASW ${ }^{\mathrm{TM}}$ Statistics 18 (SPSS Inc. 233, Chicago, IL, Estados Unidos).

\section{Consideraciones éticas}

Se buscó causar el mínimo estrés en la recolección de las muestras y el manejo de los animales. Se contó con el consentimiento y la presencia de los dueños de cada perro, y las muestras se recolectaron de acuerdo con los protocolos y reglamentos vigentes (Norma Oficial Mexicana NOM-062-ZOO-1999, Especificaciones técnicas para la producción, el cuidado y el uso de los animales de laboratorio; Reglamento de la Ley Para la Protección de la Fauna del Estado de Yucatán).

\section{Resultados}

Se muestrearon 128 perros domésticos, de los cuales $28,1 \%(36 / 128)$ correspondía a hembras y, 71,8 \% (92/128), a machos. El $100 \%$ (128/128) de los animales era mestizo (cruce de razas) y procedía de la misma comunidad. En cuanto a las edades, 6,2 \% (8/128) correspondía a jóvenes (menores a un año de edad), $79 \%$ (101/128), a adultos (igual o mayores a un año de edad y menores a seis años de edad), y 14,8 \% (19/128), a perros de edad avanzada (seis años de edad o mayores).

La PCR anidada para el gen de $17 \mathrm{kDa}$ arrojó seis extracciones positivas. La PCR estándar (punto final) para el gen omp $B$ corroboró las extracciones positivas $y$, además, permitió identificar una muestra que no se había determinado con la PCR anidada del gen de $17 \mathrm{kDa}$, por lo que la frecuencia de infección global fue de 5,45\% (7/128). En el cuadro 1 se muestra la frecuencia de perros positivos para Rickettsia spp. según edad y sexo. De los perros positivos, 85,7 \% (6/7) correspondía

Cuadro 1. Frecuencia de perros con resultados positivos para Rickettsia spp. según sexo y edad en la comunidad rural de Bolmay, Yucatán, México

\begin{tabular}{lrcc}
\hline Variables & Total & Positivos & Frecuencia (\%) \\
\hline Sexo & & & \\
Macho & 92 & 6 & 6,5 \\
Hembra & 36 & 1 & 2,7 \\
Edad & & & \\
$\quad$ Adulto & 101 & 5 & 4,9 \\
Edad avanzada & 19 & 2 & 10,5 \\
Joven & 8 & 0 & 0 \\
\hline
\end{tabular}

a machos $y, 14,2 \%(1 / 7)$, a hembras. La frecuencia de infectados por edades fue de $71,4 \%(5 / 7)$ en adultos y de $28,5 \%(2 / 7)$ en perros de edad avanzada; no se encontró infección en ninguno de los perros menor de un año.

El análisis de alineamiento de los amplicones positivos en el GenBank mostró homologías de 100 y $99 \%$ con los fragmentos de los genes omp $B$ y de 17 kDa de $R$. typhi, respectivamente (números de acceso: KJ648944.1 y JX198507.1). En la prueba de ji al cuadrado no se encontró asociación entre los animales infectados y las variables estudiadas $(p>0,05)$.

\section{Discusión}

Hasta donde sabemos, este es el primer estudio que reporta la infección por $R$. typhi en perros de México mediante el uso de herramientas moleculares. Se ha reportado que los perros actúan como reservorios u huéspedes de numerosas especies de Rickettsias del grupo de las fiebres manchadas y del grupo tifus (20-23), incluida $R$. typhi. La detección mediante métodos serológicos (24-29) y moleculares (7) de la infección por $R$. typhi en perros, y su potencial riesgo para el mantenimiento y la transmisión del tifus múrido, se han reportado en diversos estudios. Los perros se infectan con R. typhi por su convivencia con los gatos y sus pulgas (Ctenocephalides felis), descritas como el vector de $R$. typhi, las cuales también infestan a los perros (7).

Las frecuencias reportadas en los estudios serológicos fluctúan entre 0,4\% (25) y 26,9 \% (26), y aunque en principio las técnicas utilizadas en dichos estudios no son compatibles con las de este, en los primeros se reportaron porcentajes de infección superiores e inferiores en comparación con la frecuencia de infección reportada en esta investigación (5,5\%). Por otra parte, en el estudio de Nogueras, et al. (7), llevado a cabo en España utilizando PCR, la frecuencia de infección global reportada $(4,5 \%)$ fue ligeramente inferior a la encontrada en el presente estudio. Estas diferencias pueden explicarse, entre otros factores, por las variantes climatológicas que prevalecen en las regiones, así como por la resistencia a la infección del huésped y las preferencias alimenticias del vector $(7,30)$. Asimismo, se ha reportado que el tiempo de circulación de $R$. typhi en el torrente circulatorio de los perros es breve (7), lo cual también puede influir en los porcentajes de infección reportados en estudios que utilizan pruebas diagnósticas directas como el presente. 
Rickettsia typhi se encuentra circulando de manera natural en la comunidad de estudio, lo cual implicaría un riesgo potencial de transmisión del tifus múrido a los seres humanos en forma de brote. El papel del perro como reservorio $u$ huésped de $R$. typhi en la comunidad estudiada, se ve corroborado por los casos seropositivos de tifus múrido detectados previamente en esta comunidad (Secretaría de Salud, datos sin publicar).

Además de proporcionar evidencia molecular de la presencia de $R$. tyhi en perros de la comunidad, los resultados del estudio resaltan la importancia de usar estos animales como centinelas en la detección del tifus múrido y otras rickettsias $(31,32)$. Asimismo, se confirmó que los perros pueden actuar como enlace entre el ciclo doméstico y el peridoméstico (33), debido a que en las comunidades rurales de Yucatán estos animales se mueven libremente en ambos ambientes $y$, en numerosas ocasiones, sirven de vehículo de ectoparásitos vectores de especies de Rickettsia (31) que se infectan cuando se presenta la bacteriemia en los perros huéspedes (7).

En el presente estudio no se encontró asociación entre la edad, el sexo, la raza, y la procedencia de los perros muestreados, y el resultado de la prueba molecular. Esto pudo deberse a la baja frecuencia de infección encontrada, a pesar de que en los perros adultos esta fue mayor que en los perros de edad avanzada $(71,42 \%$ Vs. $28,5 \%)$, lo que probablemente sea consecuencia de un mayor contacto con ectoparásitos infectados $(31,34)$.

Este estudio demostró que los perros participan en el ciclo de infección de $R$. typhi en la región. No obstante, en futuras investigaciones se recomienda incluir las especies de artrópodos vectores, así como el estudio del potencial patógeno de $R$. typhi en reservorios y huéspedes.

\section{Agradecimientos}

A los Servicios de Salud de Yucatán, por facilitar la recolección de las muestras. Al personal de la Unidad Colaborativa de Bioensayos Entomológicos del Campus de Ciencias Biológicas y Agropecuarias, y al Laboratorio de Enfermedades Emergentes y Reemergentes del Centro de Investigaciones Regionales "Dr. Hideyo Noguchi" de la Universidad Autónoma de Yucatán.

\section{Conflicto de intereses}

Los autores declaran que no existe ningún conflicto de intereses para la publicación del presente manuscrito.

\section{Financiación}

Este estudio forma parte del proyecto "Estudio multidisciplinario para la identificación de variables asociadas a la transmisión de enfermedades zoonóticas y enfermedades transmitidas por vector en Yucatán", de la Red Epidemiológica de Enfermedades Zoonóticas y Transmitidas por Vector de Importancia en Salud Pública (PROMEP 2008-103.5/09/12.58; UADY-SISTPROY CIRB2009-0006).

\section{Referencias}

1. Raoult D, Roux V. Rickettsioses as paradigms of new or emerging infectious diseases. Clin Microbiol Rev. 1997; 10:694-719.

2. Milagres BS, Padilha AF, Padilha AF, Montandon CE, Freitas RN, Pacheco R, et al. Spotted fever group rickettsia in small rodents from areas of low endemicity for Brazilian spotted fever in the eastern region of Minas Gerais State, Brazil. Am J Trop Med Hyg. 2013;88:937-9. http://dx.doi. org/10.4269/ajtmh.12-0609

3. Raoult D, Woodward T, Dumler JS. The history of epidemic typhus. Infec Dis Clin North Am. 2004;18:127-40. http:// dx.doi.org/10.1016/S0891-5520(03)00093-X

4. Parola P, Paddock CD, Raoult D. Tick-borne rickettsioses around the world: Emerging diseases challenging old concepts. Clin Microbiol Rev. 2005;18:719-56. http://dx.doi. org/10.1128/CMR.18.4.719-756.2005

5. Wall R. A Ticking clock for tickborne disease? Vet Rec. 2012;170:326-8. http://dx.doi.org/10.1136/vr.e2158

6. Rovery C, Brouqui P, Raoult D. Questions on Mediterranean spotted fever a century after its discovery. Emerg Infect Dis. 2008;14:1360-7. http://dx.doi.org/10.3201/eid1409.071133

7. Nogueras MM, Pons I, Pla J, Ortuño A, Miret J, Sanfeliu I, et al. The role of dogs in the eco-epidemiology of Rickettsia typhi, etiological agent of murine typhus. Vet Microbiol. 2013;163:97-102. http://dx.doi.org/10.1016/j. vetmic.2012.11.043

8. Breitschwerdt EB, Meuten DJ, Walker DH, Levy M, Kennedy K, King M, et al. Canine Rocky Mountain spotted fever: A kennel epizootic. Am J Vet Res. 1985;46:2124-8.

9. Nicholson WL, Allen KE, McQuiston JH, Breitschwerdt EB, Little SE. The increasing recognition of rickettsial pathogens in dogs and people. Trends Parasitol. 2010;26: 205-212. http://dx.doi.org/10.1016/j.pt.2010.01.007

10. Adjemian J, Parks S, McElroy K, Campbell J, Eremeeva ME, Nicholson WL, et al. Murine typhus in Austin, Texas, USA, 2008. Emerg Infect Dis. 2010;16:412-7. http://dx.doi. org/10.3201/eid1603.091028

11. Zavala-Castro JE, Zavala-Velázquez JE, Sulú-Uicab JE. Murine typhus in child, Yucatán, México. Emerg Infect Dis. 2009;15:972-4. http://.dx.doi.org/10.3201/eid1506.081367

12. Dzul-Rosado K, González-Martínez P, Peniche-Lara G, Zavala-Velázquez J, Zavala-Castro J. Murine typhus in humans, Yucatan, Mexico. Emerg Infect Dis. 2013;19: 1021-2. http://dx.doi.org/10.3201/eid1906.121400 
13. Zavala-Castro JE, Dzul-Rosado KR, Peniche-Lara G, Tello-Martín R, Zavala-Velázquez JE. Isolation of Rickettsia typhi from Human, México. Emerg Infect Dis. 2014;20:1411-2. http://dx.doi.org/10.3201/eid2008.130095

14. Peniche-Lara G, Dzul-Rosado K, Pérez-Osorio C, Zavala-Castro J. Rickettsia typhi in rodents and $R$. felis in fleas in Yucatán, as a possible causal agent of undefined febrile cases. Rev Inst Trop Sao Paulo. 2015;57:129-32. http://dx.doi.org/10.1590/S0036-46652015000200005

15. Instituto Nacional de Estadística y Geografía. Recursos naturales. Fecha de consulta: 3 de mayo de 2015. Disponible en: http://www.inegi.org.mx/geo/contenidos/recnat/default. aspx.

16. Choi YJ, Jang WJ, Ryu JS, Lee SH, Park KH, Paik HS, et al. Spotted fever group and typhus group rickettsioses in humans, South Korea. Emerg Infect Dis. 2005;11:237-44.

17. Webb L, Carl M, Malloy DC, Dasch GA, Azad AF. Detection of murine typhus in fleas by using the polimerase chain reaction. J Clin Microb. 1990;28:530-4.

18. Schriefer ME, Sacci JB Jr, Dumler JS, Bullen MG, Azad AF. Identification of a novel rickettsial infection in a patient diagnosed with murine typhus. J Clin Microbiol.1994;32: 949-54.

19. Peniche-Lara G, Zavala-Velázquez J, Dzul-Rosado K, Walker DH, Zavala-Castro JE. Simple method to differentiate among Rickettsia species. J Mol Microbiol Biotechnol. 2013;23:203-8. http://dx.doi.org/10.1159/ 000348298

20. Hii SF, Kopp SR, Abdad MY, Thompson MF, O'Leary CA, Rees RL, et al. Molecular evidence supports the role of dogs as potential reservoirs for Rickettsia felis. Vector Borne Zoonotic Dis. 2011;11:1007-12. http://dx.doi.org/10.1089/ vbz.2010.0270

21. Kamani J, Baneth G, Mumcuoglu KY, Waziri NE, Eyal O, Guthmann Y, et al. Molecular detection and characterization of tick-borne pathogens in dogs and ticks from Nigeria. PLos Negl Trop Dis. 2013;7:e2108. http://dx.doi.org/10.1371/ journal.pntd.0002108

22. Barrett A, Little SE, Shaw E. "Rickettsia amblyommir" and R. montanensis infection in dogs following natural exposure to ticks. Vector Borne Zoonotic Dis. 2014;14:20-5. http:// dx.doi.org/10.1089/vbz.2013.1325

23. Solano-Gallego L, Caprì A, Pennisi MG, Caldin M, Furlanello T, Trotta M. Acute febrile illness is associated whit Rickettsia spp. infection in dogs. Parasit Vectors. 2015;10:216. http://dx.doi.org/10.1186/s13071-015-0824-3
24. Huxsoll DL, Shirai A, Robinson DM, Yap LF, Lim BL. Presence of antibodies to scrub typhus and murine typhus in dogs from Selangor, Peninsular Malaysia. Southeast Asian J Trop Med Public Health. 1977;8:232-5.

25. Soliman AK, Botros BA, Ksiazek TG, Hoogstraal H, Helmy I, Morrill JC. Seroprevalence of Rickettsia typhi and Rickettsia conorii infection among rodents and dogs in Egypt. J Trop Med Hyg. 1989;92:345-9.

26. Bacellar F, Dawson JE, Silveira CA, Filipe AR. Antibodies against Rickettsiaceae in dogs of Seúbal, Portugal. Cent Eur J Public Health. 1995;3:100-2.

27. Lledó L, Gegúndez MI, Serrano JL, Saz JV, Beltrán M. A sero-epidemiological study of Rickettsia typhi infection in dogs from Soria province, central Spain. Ann Trop Med Parsitol. 2003;97:861-4.

28. Nogueras MM, Pons I, Ortuño A, Segura F. Seroprevalence of Rickettsia typhi and Rickettsia felis in dogs from northeastern Spain. Clin Microbiol Infect. 2009;15:237-8. http:// dx.doi.org/10.1111/j.1469-0691.2008.02156

29. Nanayakkara DM, Rajapakse RP, Wickramasinghe S, Kularatne SA. Serological evidence for exposure of dogs to Rickettsia conorii, Rickettsia typhi and Orientia tsutsugamushi in Sri Lanka. Vector Borne Zoonotic Dis. 2013;13:545-9. http://dx.doi.org/10.1089/vbz.2012.1049

30. Espejo E, Alegre MD, Font B, Font A, Segura F, Bella F. Antibodies to Rickettsia conorii in dogs: Seasonal differences. Eur J Epidemiol. 1993;9:344-6.

31. Tomassone L, Conte V, Parrilla G, De Meneghi D. Rickettsia infection in dogs and Rickettsia parkeri in Amblyomma tigrinum ticks, Cochabamba Department, Bolivia. Vector Borne Zoonotic Dis. 2010;10:953-8. http:// dx.doi.org/10.1089/vbz.2009.0126

32. Breitschwerdt EB, Moncol DJ, Corbett WT, MacCormack JN, Burgdorfer W, Ford RB, et al. Antibodies to spotted fever-group Rickettsiae in dogs in North Carolina. Am J Vet Res. 1987 48:1436-40.

33. Nicholson WL, Allen KE, McQuiston JH, Breitschwerdt EB, Little SE. The increasing recognition of rickettsial pathogens in dogs and people. Trends Parasitol. 2010;26: 205-12. http://dx.doi.org/10.1016/j.pt.2010.01.007

34. Pinter A, Horta MC, Pacheco RC, Moraes-Filho J, Labruna MB. Serosurvey of Rickettsia spp. in dogs and humans from an endemic area for Brazilian spotted fever in the State of São Paulo, Brazil. Cad Saúde Pública. 2008;24:247-52. http://dx.doi.org/10.1590/S0102-311X200 8000200003 